\title{
Cross-protective mucosal immunity mediated by memory Th17 cells against Streptococcus pneumoniae lung infection
}

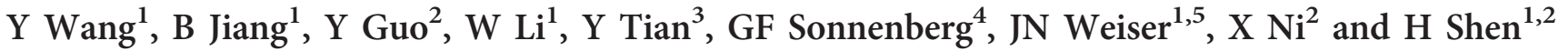

Pneumonia caused by Streptococcus pneumoniae $(S p)$ remains a leading cause of serious illness and death worldwide. Immunization with conjugated pneumococcal vaccine has lowered the colonization rate and consequently invasive diseases by inducing serotype-specific antibodies. However, many of the current pneumonia cases result from infection by serotype strains not included in the vaccine. In this study, we asked if cross-protection against lung infection by heterologous strains can be induced, and investigated the underlying immune mechanism. We found that immune mice recovered from a prior infection were protected against heterologous $\mathrm{Sp}$ strains in the pneumonia challenge model, as evident by accelerated bacterial clearance, reduced pathology, and apoptosis of lung epithelial cells. Sp infection in the lung induced strong T-helper type 17 (Th17) responses at the lung mucosal site. Transfer of CD4 ${ }^{+}$Tcells from immune $^{-17}$ mice provided heterologous protection against pneumonia, and this protection was abrogated by interleukin-17A (IL17A) blockade. Transfer of memory CD4 ${ }^{+}$Tcells from IL-17A-knockout mice failed to provide protection. These results indicate that memory Th17 cells had a key role in providing protection against pneumonia in a serotype-independent manner and suggest the feasibility of developing a broadly protective vaccine against bacterial pneumonia by targeting mucosal Th17 T cells.

\section{INTRODUCTION}

Streptococcus pneumoniae $(S p)$ is a Gram-positive bacterium that is frequently found asymptomatically colonizing the mucosal surface of the human nasopharynx. Colonization rates are as high as 50\% in infants and $5-10 \%$ in healthy adult populations. ${ }^{1}$ High colonization rates in young children are the primary source of pneumococcal transmission through direct contact or by aerosols. ${ }^{2}$ This ability of $S p$ to establish asymptomatic colonization is an important mechanism that the organism uses to keep itself widely distributed in humans. ${ }^{2-4}$ Although the interactions between $S p$ and humans in the upper respiratory tract (RT) are benign, certain conditions (such as influenza virus infections) can alter the balance of the host- $S p$ interaction, leading to the progression of $S p$ infections into deep tissues and the development of various diseases, including otitis media, pneumonia, sepsis, and meningitis. ${ }^{5-7} S p$ is the most frequent cause of secondary bacterial pneumonia following influenza virus infection that often leads to severe diseases requiring hospitalization and resulting in high mortality. ${ }^{6,7}$

$S p$ bacteria are encapsulated by polysaccharide layers surrounding their outer cell walls. The polysaccharide capsule has a significant role in the virulence of the organism, functioning to reduce clearance by mucosal secretions, prevent bacterial uptake by phagocytes, and decrease the binding of complement to the bacterial surface. ${ }^{8,9}$ The polysaccharide capsule is also a major target of antibody responses, and to date over 90 distinct serotypes of $S p$ have been identified that are characterized by structural and compositional variations in their capsules. Current pneumococcal-conjugated vaccine (PCV) is based on capsular polysaccharides conjugated to a

\footnotetext{
${ }^{1}$ Department of Microbiology, Perelman School of Medicine, University of Pennsylvania, Philadelphia, Pennsylvania, USA. ${ }^{2}$ Beijing Pediatric Research Institute, Beijing Children's Hospital, Capital Medical University, Beijing, China. ${ }^{3}$ Department of Pharmacology, Center for Translational Medicine, Temple University School of Medicine, Philadelphia, Pennsylvania, USA. ${ }^{4}$ Joan and Sanford I Weill Department of Medicine, Division of Gastroenterology and Hepatology, Department of Microbiology and Immunology, and The Jill Robert's Institute for Research in Inflammatory Bowel Disease, Weill Cornell Medical College, New York, New York, USA and ${ }^{5}$ Department of Microbiology, NYU School of Medicine, New York, New York, USA. Correspondence: H Shen (hshen@mail.med.upenn.edu)
} 
protein carrier and its introduction has resulted in marked reductions in rates of nasopharyngeal colonization and consequently invasive pneumococcal diseases. However, PCV-induced immunity is antibody-based and highly serotype-specific, and only protective against 13 serotype strains whose capsular polysaccharide types are included in PCV. ${ }^{10,11}$ In recent years, there is increased prevalence of pneumococcal diseases caused by serotypes that are not included in PCV-a phenomenon termed serotype replacement that is leading to a decline of PCV efficacy in clinics. ${ }^{12,13}$ In addition, the high cost of PCV due to complex manufacturing processes limits its use in developing countries. Thus, there is an urgent, global need for an alternative pneumococcal vaccine that is affordable and can provide broad protection against various circulating serotype strains., ${ }^{2,14-17}$

The development of mouse models of $S p$ colonization and invasive disease has aided in the identification of bacterial factors involved in Sp pathogenesis and host immune mechanisms of protection. Asymptomatic colonization (a carrier state) can be established by a concentrated bacterial inoculum applied in a small $(10 \mu \mathrm{l})$ volume to the nares of unanesthetized mice. In this model of defined nasal infection, bacteria remain detectible in nasal lavage fluid for several weeks without progressing into invasive diseases. ${ }^{18,19}$ Invasive $S p$ infections can be experimentally induced by the intranasal administration of a high volume bacterial inoculum (30-50 $\mu$ l) to anesthetized mice (direct lung infection model). Dosage in this manner bypasses the initial colonization step and introduces the organism directly into the lower RT, whereby pneumonia rapidly develops. ${ }^{20}$ Prior colonization can induce protection against subsequent direct lung challenge with the same serotype strains (homologous), and the homologous protection against pneumonia is dependent on antibodies ${ }^{21,22}$ and T-helper type 17 (Th17) CD4 ${ }^{+}$cells. $^{22}$ However, prior colonization induces minimal or partial protection against pneumonia by different serotype strains (heterologous). ${ }^{23}$ Repeated immunizations with killed whole-cell vaccines or conserved proteins with potent experimental adjuvants (pertussis toxin) confer protection against lung infections by different serotype strains. ${ }^{24-29}$ The mechanisms of crossprotection against pneumonia remain controversial and undefined. Depletion of B cells, $\mathrm{CD} 4^{+}$, or $\mathrm{CD}^{+}{ }^{+} \mathrm{T}$ cells after immunization did not abrogate protection. ${ }^{25,27}$ On the other hand, immunization of $\mu \mathrm{MT}$ mice with conserved $S p$ proteins/adjuvant failed to protect against pneumonia induced by heterologous strains, suggesting a role for B cells/antibodies. ${ }^{29}$

To study the immune mechanism of cross-protection against pneumonia, we first tested approaches to induce the highest level of protection using the mouse model of direct lung infection. ${ }^{21,23,30}$ We found that a sublethal prior lung infection provided the best protection against pneumonia from subsequent direct lung infection by a heterologous $S p$ strain, whereas colonization or immunization with heat-killed $S p$ provided little or partial protection. We further investigated the immune mechanism of cross-protection induced by a prior sublethal lung infection. Our results showed that Th17 cells, not antibodies, from immune mice contributed to the bacterial clearance in the lung following challenge with several different serotype strains.

\section{RESULTS}

\section{Prior lung infection induces cross-protection in the murine pneumonia model}

To test if cross-protection against pneumonia can be induced, we immunized B6 mice by colonization, lung infection, or intrapulmonary immunization with heat-killed $S p$ and asked if these mice are protected against subsequent direct lung infection by a heterologous $S p$ strain. For colonization, mice were inoculated intranasally with $1 \times 10^{7}$ colony-forming units (CFU) of P1121 (serotype 23F) in $10 \mu$ without anesthesia, which resulted in colonization of the upper RT. ${ }^{31}$ For direct lung infections, anesthetized mice were inoculated intranasally with $1 \times 10^{7} \mathrm{CFU}$ of P1121 in $30 \mu \mathrm{l}$, which resulted in direct infection of the lower RT and acute bacterial pneumonia. Similarly, $1 \times 10^{7} \mathrm{CFU}$ of heat-killed P1121 were introduced intranasally to the lung of B6 mice in $30 \mu$ under anesthesia (intrapulmonary immunization). After 30 days, these mice were challenged with a lethal dose $\left(2 \times 10^{7} \mathrm{CFU}\right)$ of $S p$ strain TIGR4 (T4, serotype 4) by direct lung infection that resulted in acute pneumonia and mortality in naïve mice. Mice previously colonized with P1121 all succumbed to T4 challenge, although they survived for 1-2 days longer than the unimmunized mice. Immunization with heat-killed $\mathrm{P} 1121$ provided partial protection against T4 challenge, with $50 \%$ survival. Mice that resolved a prior lung infection by $\mathrm{P} 1121$ were $90 \%$ protected against subsequent challenge with the heterologous T4 strain (Figure 1a). These results show that prior live lung infection induces the highest level of protective immunity against pneumonia by a heterologous strain.

To further characterize heterologous protection induced by prior lung infection, we challenged mice with a lower dose of T4 $\left(5 \times 10^{6} \mathrm{CFU}\right)$ that caused morbidity but not death, allowing us to assess morbidity, bacterial load, and lung pathology at different time-points after the challenge. Mice resolved a prior P1121 lung infection (P1121 immune) and control mice lost body weight at a similar rate during the first two days after the T4 challenge. However, P1121-immune mice regained body weight much faster than control mice and fully recovered to their original body weight around days 7-9 postinfection (Figure 1b). Bacterial loads in the lung and bronchoalveolar lavage fluid (BALF) were determined at days $0.5,1,2,3,5$, and 7 after T4 challenge. By day 1 after T4 challenge, there were already significantly fewer bacteria $(\sim 100$-fold) in the lungs and BALF of P1121-immune mice compared with unimmunized control mice. P1121-immune mice cleared bacteria between 3 and 5 days after infection, which was faster than unimmunized mice that did not clear till day 7 (Figure 1c,d). Histology showed that P1121-immune mice had moderate lung pathology with fewer inflammatory cells and less apoptosis of bronchial epithelial cells compared with control mice (Figure 1e). Taken together, these results of 


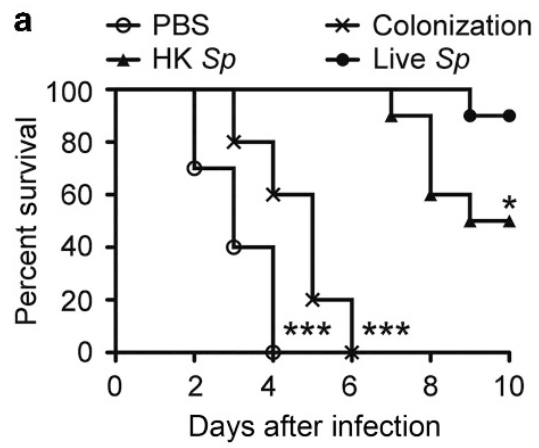

b

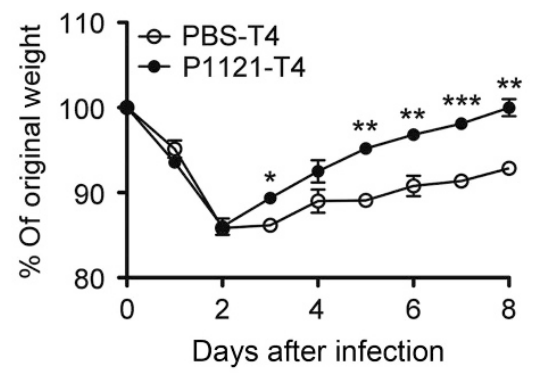

C

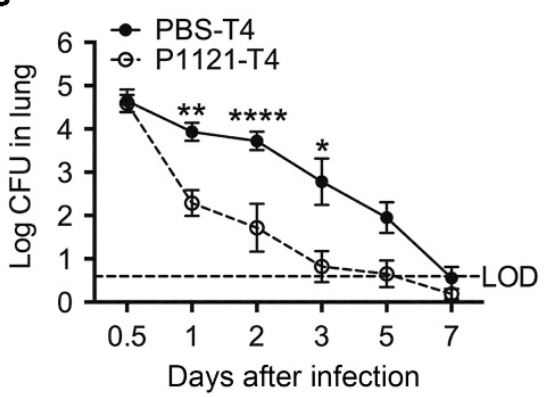

d
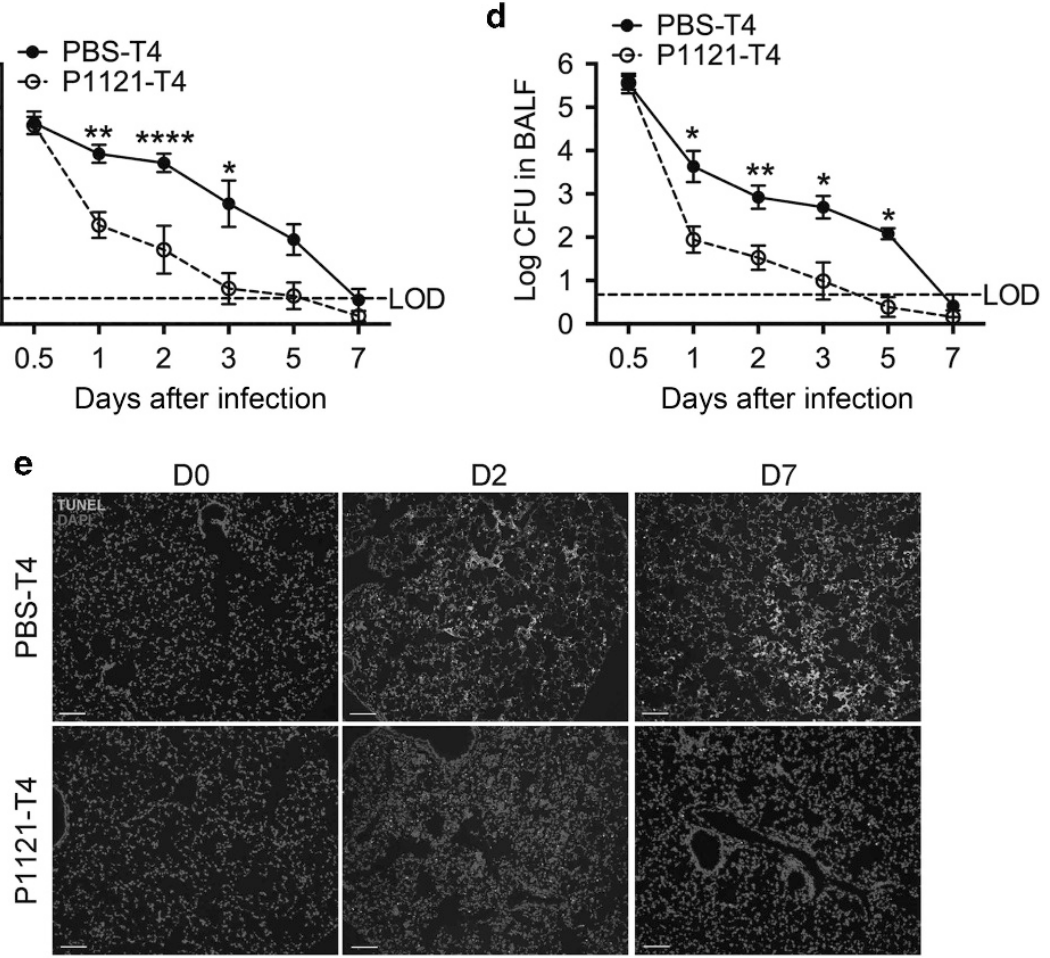

Figure 1 Cross-protection against pneumonia induced by prior colonization, lung infection, or intrapulmonary immunization with heat-killed Sp. Mice were colonized with P1121 (colonization), infected with a sublethal dose of P1121 (live Sp), or immunized with heat-killed P1121 (HK Sp), and 30 days later were challenged by direct lung infection with a (a) lethal or (b-d) sublethal dose of T4. (a) Survival rates; (b) body weight loss; bacterial loads in (c) lung homogenate and (d) bronchoalveolar lavage fluid (BALF) on different days after T4 challenge; (e) representative TUNEL (terminal deoxynucleotidyl transferase dUTP nick-end labeling) (green) and DAPI (4',6-diamidino-2-phenylindole; blue) staining of lung section at indicated days after T4 challenge of P1121 immune (P1121-T4) and control (PBS-T4) mice. Data are mean \pm s.e.m. from at least three independent experiments with $>10$ mice in each group. Bar $=200 \mu \mathrm{m}$. LOD, limit of detection. A full color version of this figure is available at the Mucosal Immunology journal online.

reduced morbidity and mortality, lower bacterial loads, and less lung pathology clearly show that prior lung infection induces protective immunity against subsequent lung infection by a heterologous strain.

\section{$\mathrm{CD}^{+}{ }^{+} \mathrm{T}$ cells mount a robust Th17 primary and recall responses localized in the lung mucosa following $S p$ infection}

To understand the immune mechanisms of cross-protection against pneumonia, we examined the primary responses in T4-infected mice (T4) as well as recall responses in P1121immune mice challenged with T4 (P1121-T4). Compared with naïve mice, T4-infected mice had much higher percentages and numbers of $\mathrm{CD} 4{ }^{+} \mathrm{T}$ cells in the lungs. The P1121-T4 mice had even higher percentages and numbers of $\mathrm{CD} 4^{+} \mathrm{T}$ cells in the lungs than T4-infected mice (Figure 2a,b). Most of CD4 ${ }^{+}$ $\mathrm{T}$ cells in the lungs of T4-infected and P1121-T4 mice had an activated $\mathrm{CD} 44^{\text {high }}$ phenotype (data not shown). On the other hand, lung $\mathrm{CD}^{+}{ }^{+} \mathrm{T}$-cell population was not significantly increased in T4 and P1121-T4 mice compared with naïve mice (Figure 2a,b). Furthermore, no significant differences were observed in both $\mathrm{CD} 4{ }^{+}$and $\mathrm{CD} 8{ }^{+} \mathrm{T}$ cells in the spleen of naïve, T4, and P1121-T4 groups (Supplementary Figure S1A,B online). These results show that $S p$ lung infection induces a strong $\mathrm{CD} 4{ }^{+} \mathrm{T}$-cell response localized in the lung mucosa, and immune mice mount an even stronger $\mathrm{CD} 4^{+}$ 
a
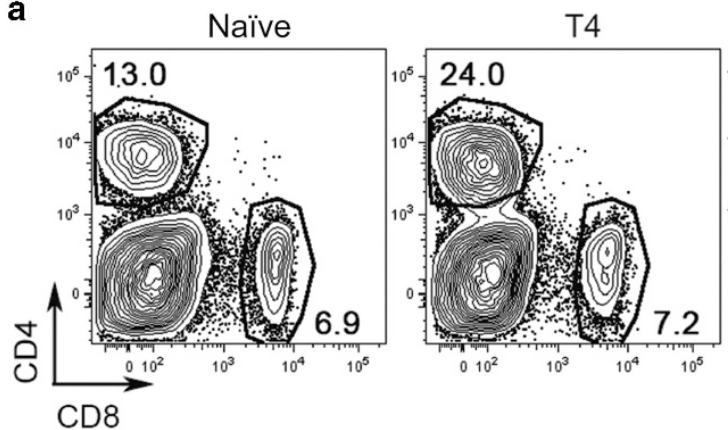

C

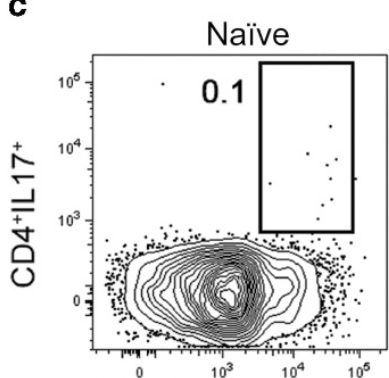

c
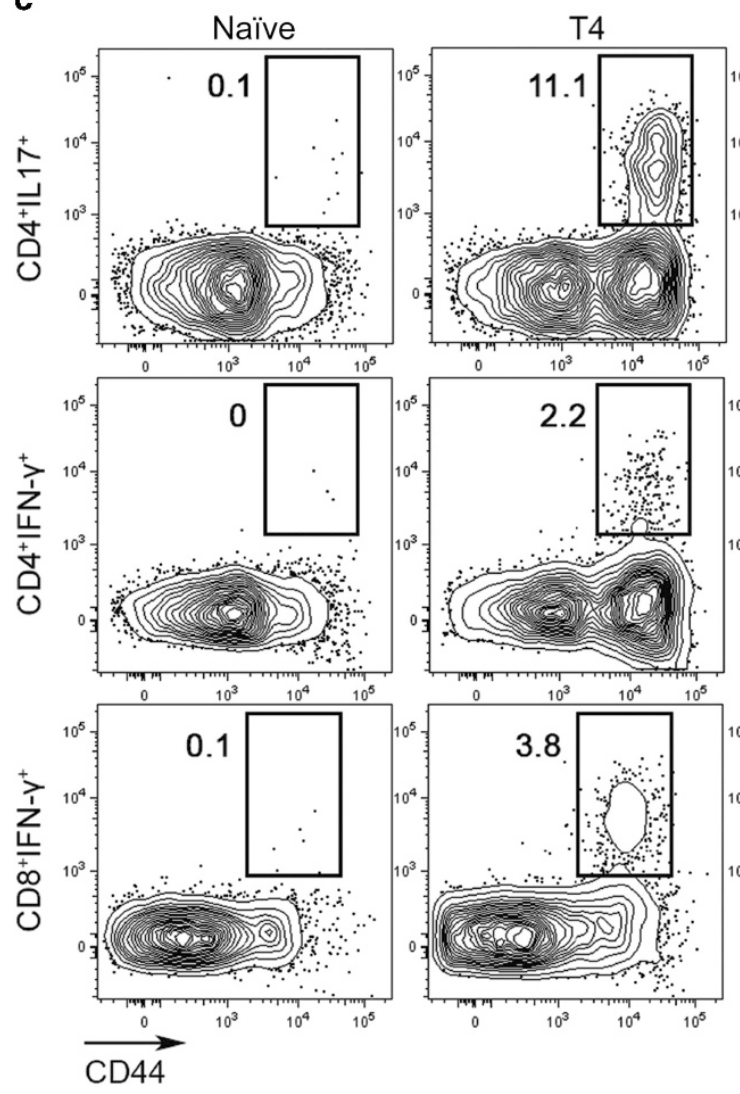

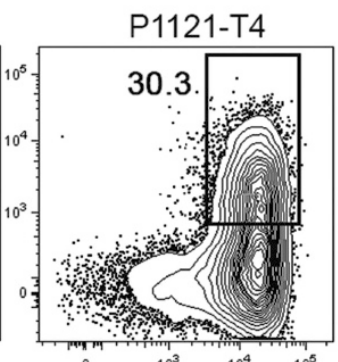

P1121-T4
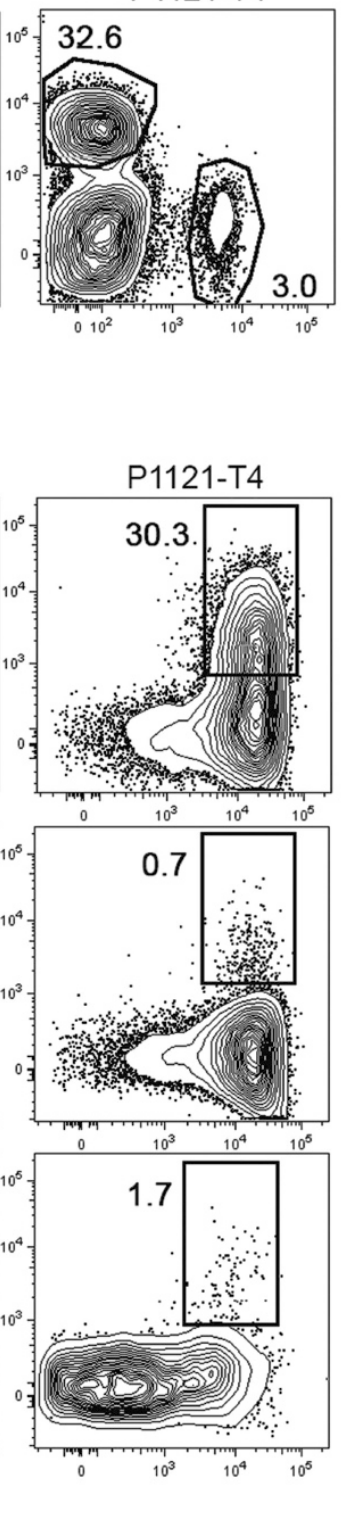

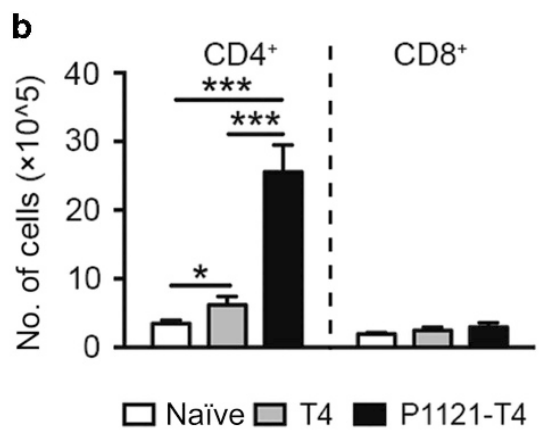

d

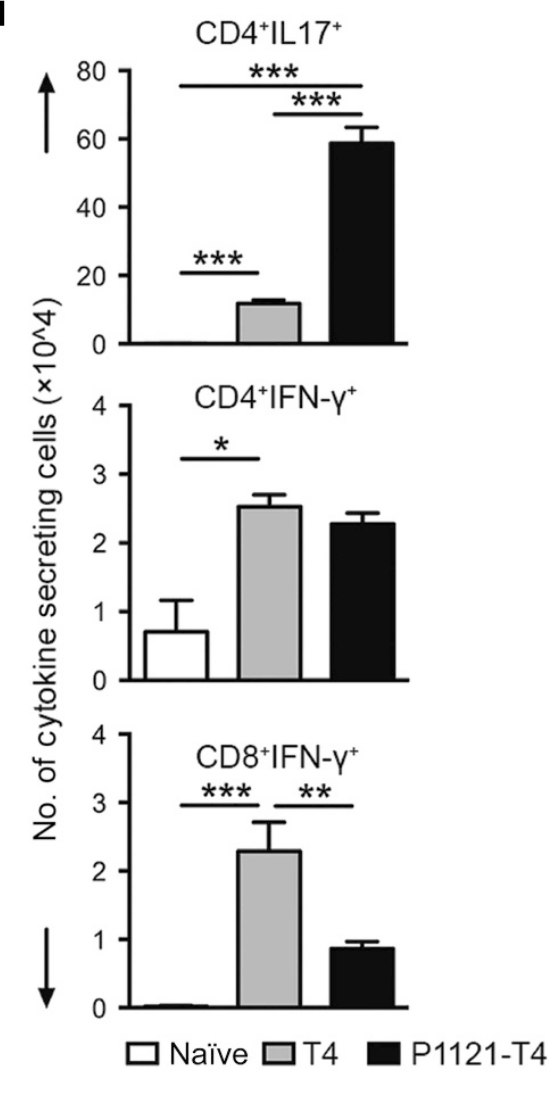

Figure $2 \mathrm{CD}^{+} \mathrm{T}$ cells mount a rapid and robust T-helper type 17 (Th17) recall responses in the lung. (a) Percentage and (b) absolute number of pulmonary CD4 and CD8 T cells in naïve mice, mice infected with T4 only (T4), or P1121-immune mice challenged with T4 (P1121-T4) on day 7 after T4. Interleukin-17A (IL-17A) and interferon- $\gamma\left(\right.$ IFN- $\gamma$ ) production by CD4 ${ }^{+}$and CD8 ${ }^{+}$T cells after stimulation with heat-killed T4 as (c) visualized by FACS and (d) calculated as the number of $\mathrm{CD} 4^{+} \mathrm{IL}-17 \mathrm{~A}^{+}, \mathrm{CD} 4^{+} \mathrm{IFN}-\gamma^{+}$, and $\mathrm{CD} 8^{+} \mathrm{IFN}-\gamma^{+}$per lung.

T-cell response, presumably due to recall responses by memory $\mathrm{CD}^{+}{ }^{+} \mathrm{T}$ cells.

To characterize the function of $\mathrm{T}$ cells responding to $S p$ infection, we analyzed cytokine expression by intracellular cytokine staining following in vitro stimulation with heat-killed T4. A high percentage $(\sim 11 \%)$ of CD $4^{+}$T cells from lungs of T4-infected mice expressed interleukin-17A (IL-17A), whereas a small percentage of them $(\sim 2 \%)$ produced interferon- $\gamma$ (IFN- $\gamma$ ) (Figure 2c,d). In the spleen, few CD $4^{+} \mathrm{T}$ cells expressed either IL-17A $(\sim 0.3 \%)$ or IFN- $\gamma(\sim 0.5 \%)$ (Supplementary Figure S1C,D). Lung CD ${ }^{+}$T cells expressed only IFN- $\gamma(\sim 4 \%$ in T4-infected mice; Figure 2) but no IL-17A (data not shown).
Very low levels of IFN- $\gamma^{+} \mathrm{CD}{ }^{+}$T cells were detected in the spleen of naïve and T4-infected mice (Supplementary Figure S1C). Thus, the primary T-cell response to $S p$ infection of the lung is a strong $\mathrm{CD}^{+}{ }^{+}$response localized in the lung and consisted of mostly IL-17A-secreting CD4 ${ }^{+}$T cells with a small population of IFN- $\gamma^{+} \mathrm{CD} 4{ }^{+} \mathrm{T}$ cells.

In the P1121-T4 mice, there was an even stronger Th17 response $\left(\sim 30 \% \mathrm{CD}^{+}\right)$in the lung compared with T4-infected mice $\left(\sim 11 \% \mathrm{CD}^{+}\right)$. This represented a sixfold increase in the total number of IL-17A ${ }^{+} \mathrm{CD} 4{ }^{+} \mathrm{T}$ cells in the lungs of the P1121-T4 mice over T4-infected mice (Figure 2d), indicating a strong Th17 recall response by memory CD4 ${ }^{+}$ 
a

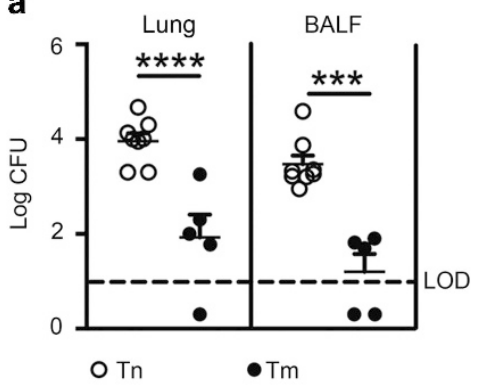

b

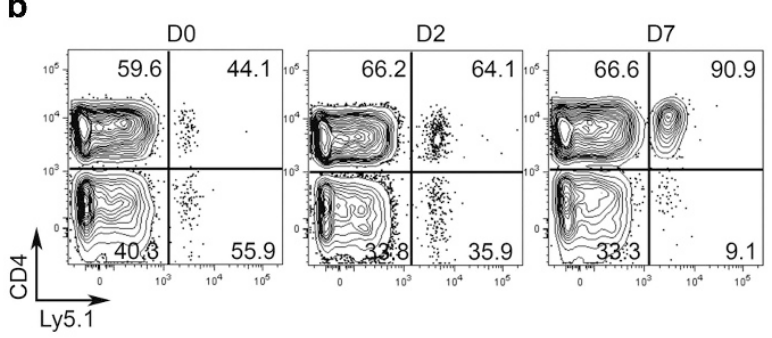

d

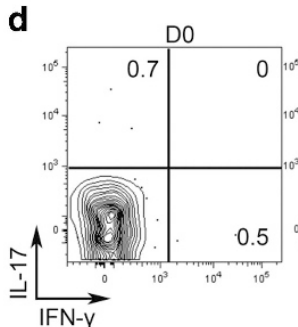

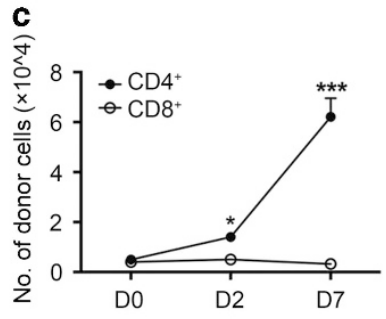

e

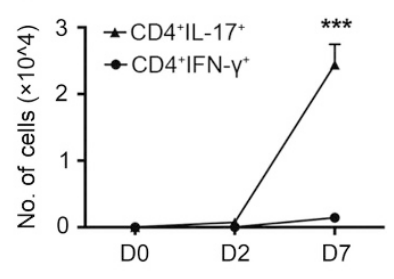

Figure 3 Transfer of Streptococcus pneumoniae (Sp)-specific memory T cells provides cross-protection against pneumonia. Lung and spleen T cells from naïve (Tn) or P1121 immune (Tm) were adoptively transferred into congenic (Ly5.2) B6 mice, which were then challenged with T4. (a) Bacterial loads in lung homogenate and bronchoalveolar lavage fluid (BALF) on day 2 after challenge. (b) Percentages and (c) number of donor (Ly5.1 ${ }^{+}$) CD4 and CD8 T cells recovered from the lung at indicated days after T4 infection. (d) Percentage and (e) number per lung of interleukin-17A (IL-17A) and interferon- $\gamma$ (IFN- $\gamma$ )-producing donor $\mathrm{CD}^{+}{ }^{+} \mathrm{T}$ cells after stimulation with heat-killed T4.

T cells. Surprisingly, there was minimal Th1 memory recall response. In fact, IFN- $\gamma^{+} \mathrm{CD} 4^{+} \mathrm{T}$ cells in the lung of P1121$\mathrm{T} 4$ mice were lower $\left(\sim 0.7 \% \mathrm{CD}^{+}\right)$compared with T4-infected mice $\left(\sim 2.2 \% \mathrm{CD}^{+}\right)$. Similarly, there were less IFN $-\gamma^{+} \mathrm{CD}^{+}{ }^{+} \mathrm{T}$ cells in the lungs of P1121-T4 mice $(\sim 1.7 \%$ $\left.\mathrm{CD} 8^{+}\right)$than in T4-infected mice $\left(\sim 3.8 \% \mathrm{CD}^{+}\right.$; Figure $\left.2 \mathbf{c}, \mathbf{d}\right)$.

The above results show that a primary $\mathrm{T} 4$ infection induces a rapid and robust $\mathrm{CD} 4{ }^{+} \mathrm{T}$-cell response in the lungs that consists of predominantly Th17 but also Th1 cells. In P1121-immune mice, the recall response to T4 challenge is exclusively by Th17 $\mathrm{CD} 4{ }^{+} \mathrm{T}$ cells with minimal contribution by $\mathrm{Th} 1 \mathrm{CD} 4^{+}$or $\mathrm{CD} 8^{+} \mathrm{T}$ cells. Taken together, these data suggest that Th17 recall response by memory $\mathrm{CD} 4{ }^{+} \mathrm{T}$ cells may have an important role in cross-protection against invasive pneumococcal disease by a different serotype strain.

\section{Sp-specific memory T cells transfer acquired immunity against heterologous $\mathrm{Sp}$ pneumonia}

To investigate the role of immune cells in protection against heterologous $S p$ pneumonia, $\mathrm{CD}^{+}$(T cells) and $\mathrm{CD}^{-}{ }^{-}$(non$\mathrm{T}$ cells) lymphocytes from the spleens and lungs of P1121immune and naïve mice were transferred into congenic (Ly5.2) B6 mice, which were then challenged with a heterologous T4 strain. Mice that received memory T (Tm) cells from P1121immune mice had 107- and 186-fold fewer bacterial CFU in the lungs and BALF, respectively, compared with mice that received naïve $\mathrm{T}(\mathrm{Tn})$ cells from unimmunized mice (Figure 3a). Mice that received non- $\mathrm{T}$ cells from immune mice were not protected, with similar numbers of CFU both in the lungs and BALF compared with mice that received non-T cells from naive mice (Supplementary Figure S2). These results show that $\mathrm{CD}_{3}{ }^{+} \mathrm{T}$ cells from P1121-immune mice provide cross-protection against lung infection with a heterologous T4 strain.

The recall response and cytokine production by donor (Ly5.1) memory $\mathrm{CD} 4{ }^{+}$and $\mathrm{CD} 8^{+} \mathrm{T}$ cells were examined on days 0,2 , and 7 after T4 challenge (Figure $3 \mathbf{b}$ ). On day $0, \sim 44 \%$ of donor $\mathrm{T}$ cells were $\mathrm{CD} 4^{+}$, while $\sim 56 \%$ being $\mathrm{CD} 8^{+}$. Following the $\mathrm{T} 4$ challenge, $\mathrm{CD} 4{ }^{+} \mathrm{T}$-cell population expanded greatly to make up $\sim 64 \%$ and $\sim 91 \%$ of the donor population on days 2 and 7 , respectively. The total number of donor $\mathrm{CD} 4^{+}$ T cells increased by 12.7 -fold from $0.4 \pm 0.7 \times 10^{4} /$ lung on day 0 to $6.2 \pm 7.5 \times 10^{4} /$ lung on day 7 . Many of the responding donor $\mathrm{CD} 4{ }^{+} \mathrm{T}$ cells expressed IL-17A ( $\sim 49 \%$ on day 7$)$, while very few of them produced IFN- $\gamma^{+}(\sim 2 \%$ on day 7$)$. In contrast to $\mathrm{CD} 4^{+} \mathrm{T}$ cells, donor $\mathrm{CD} 8^{+} \mathrm{T}$-cell population did not expand much in the lung (Figure $3 \mathbf{b}$ ), and very few IFN- $\gamma$ producing $\mathrm{CD} 8{ }^{+} \mathrm{T}$ cells were detected on day 2 or 7 after T4 challenge (Supplementary Figure S3A,B). In addition, no recall responses by donor $\mathrm{CD} 4^{+}$or $\mathrm{CD} 8{ }^{+} \mathrm{T}$ cells were evident in the spleen (Supplementary Figure S3C,D).

Thus, adoptive transfer of memory $\mathrm{T}$ cells from P1121immune mice conferred cross-protection against pneumonia. Donor memory $\mathrm{CD} 4{ }^{+}$but not $\mathrm{CD} 8{ }^{+} \mathrm{T}$ cells mounted a strong recall response in the lungs, and the responding donor $\mathrm{CD} 4^{+} \mathrm{T}$ cells consisted of mostly Th17 and very few Th1 effector cells.

\section{Cross-protection against pneumonia by memory CD4 ${ }^{+}$ $T$ cells is dependent on IL-17A}

Our results have thus far shown a strong Th17 recall response by memory $\mathrm{CD} 4^{+} \mathrm{T}$ cells in immune mice correlating with cross-protection. We next investigated the role of memory $\mathrm{CD}^{+} \mathrm{T}$ cells and IL-17A in cross-protection against pneumonia by adoptive transfer of purified immune $\mathrm{CD} 4^{+}$ 
a

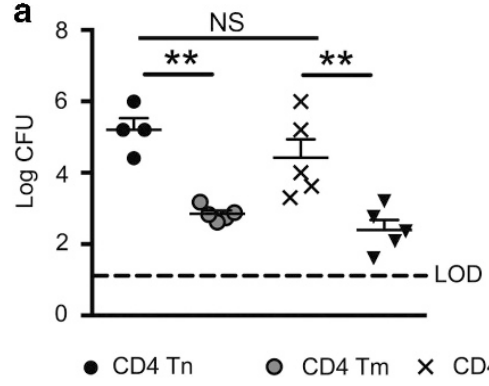

b 50

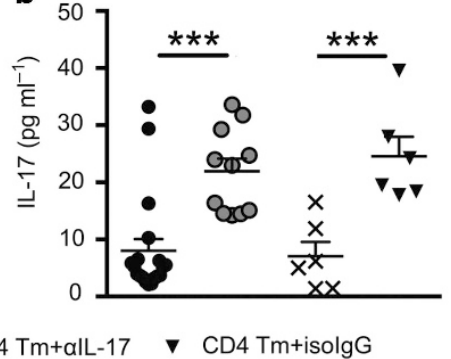

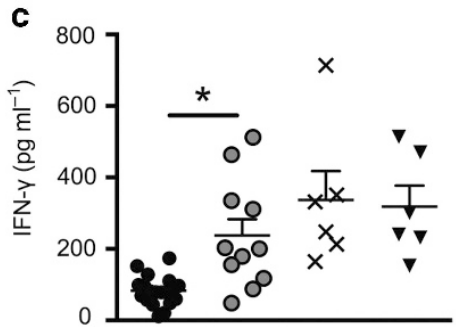

d

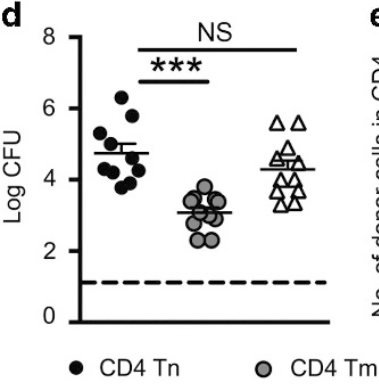

e

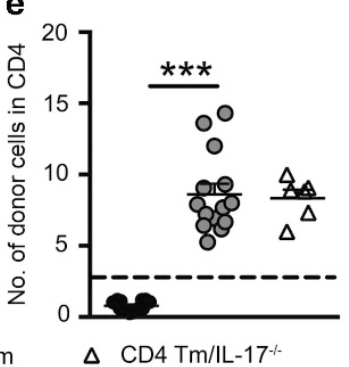

f

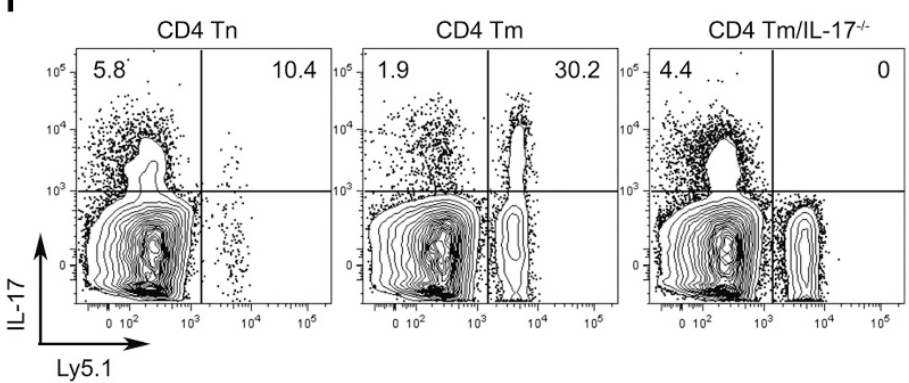

Figure 4 Memory CD4 ${ }^{+}$T cells transfer heterologous protection against pneumonia via interleukin-17 (IL-17)-mediated mechanism. (a-c) Purified $\mathrm{CD}^{+}{ }^{+}$cells from naïve $\left(\mathrm{CD} 4^{+} \mathrm{Tn}\right)$ or P1121 immune (CD4 $\left.{ }^{+} \mathrm{Tm}\right)$ were transferred into congenic (Ly5.2) B6 mice, which were then challenged with T4. One group of mice that received $\mathrm{CD} 4^{+} \mathrm{Tm}$ were also treated with IL-17-neutralizing antibody $\left(\mathrm{CD} 4^{+} \mathrm{Tm}+\alpha \mathrm{lL}-17\right)$ or isotype control antibody $\left(\mathrm{CD} 4{ }^{+} \mathrm{Tm}+\right.$ isolgG). (a) Bacterial loads in lung homogenate, (b) IL-17 and (c) interferon- $\gamma$ (IFN- $\gamma$ ) in bronchoalveolar lavage fluid (BALF) on day 2 after T4 challenge. (d-f) Purified CD4 T cells from naïve (CD4 $\left.{ }^{+} \mathrm{Tn}\right)$, P1121-immune (CD4 $\left.{ }^{+} \mathrm{Tm}\right)$, and P1121-immune IL-17 knockout (KO) mice $\left(\mathrm{CD} 4{ }^{+} \mathrm{Tm} / \mathrm{IL}-\right.$ $17^{-1-}$ ) were transferred into congenic (Ly5.2) B6 mice, which were then challenged with T4. (d) Bacterial loads in lung homogenate on day 2 after T4 challenge. (e) Percentage of donor CD4 cells in lung, and (f) IFN- $\gamma$ and IL-17A production by CD4 T cells on day 7 after T4 challenge. IgG, immunoglobulin G; Tm, memory $\mathrm{T}$; Tn, naïve $\mathrm{T}$.

T cells and in vivo blockade of IL-17A. Purified CD4 ${ }^{+}$T cells from naïve or P1121-immune mice were adoptively transferred to congenic Ly5.2-naïve recipient mice that were then challenged with T4. On day 2 after T4 challenge, mice that received memory $\mathrm{CD} 4^{+} \mathrm{T}\left(\mathrm{CD} 4^{+} \mathrm{Tm}\right)$ cells from $\mathrm{P} 1121-$ immune mice had 224-fold fewer bacteria in the lung $(1.58 \times$ $\left.10^{5} \mathrm{CFU}\right)$ than mice that received naïve $\mathrm{CD} 4^{+} \mathrm{T}\left(\mathrm{CD} 4^{+} \mathrm{Tn}\right)$ cells $\left(7 \times 10^{2} \mathrm{CFU}\right)$. Blockade of IL-17A by anti-IL-17Aneutralizing antibodies abrogated the protective effect of donor $\mathrm{CD} 4{ }^{+} \mathrm{T}$ cells, whereas isotype control immunoglobulin $\mathrm{G}$ had no effect on protection (Figure 4a). Increased levels of IL-17A and IFN- $\gamma$ were detected in BALF on day 2 after T4 challenge in mice that received $\mathrm{CD} 4{ }^{+} \mathrm{Tm}$, as compared with mice that received $\mathrm{CD} 4{ }^{+} \mathrm{Tn}$. Treatment with anti-IL-17Aneutralizing antibodies decreased the level of IL-17A in the BALF but not IFN- $\gamma$ as expected (Figure $4 \mathbf{b}, \mathbf{c}$ ).

To further evaluate a protective role for IL-17A specifically produced by memory $\mathrm{CD} 4^{+} \mathrm{T}$ cells, we purified $\mathrm{CD} 4^{+}$ T cells from IL-17 $\mathrm{A}^{-1-}$ mice that have resolved a prior P1121 infection, and adoptively transferred them into congenic naïve Ly5.2 mice. $\mathrm{CD} 4^{+} \mathrm{T}$ cells from P1121 immune IL$17 \mathrm{~A}^{-1-}$ mice $\left(\mathrm{CD} 4^{+} \mathrm{Tm} / \mathrm{IL}-17 \mathrm{~A}^{-1-}\right)$ failed to mediate cross-protection; bacterial $\mathrm{CFU}$ in mice that received $\mathrm{CD} 4^{+}$ $\mathrm{Tm} / \mathrm{IL}-17 \mathrm{~A}^{-1-}$ cells were similar to those in mice that received $\mathrm{CD}^{+}{ }^{+} \mathrm{Tn}$, while mice that received $\mathrm{CD} 4^{+} \mathrm{Tm}$ had less $\mathrm{CFU}$ (Figure 4d). Donor CD4 ${ }^{+} / \mathrm{IL}-17 \mathrm{~A}^{-1-}$ cells did not produce IL-17A as expected (Figure 4f), but they became activated and expanded to levels similar to donor $\mathrm{CD} 4{ }^{+} \mathrm{T}$ cells from immune B6 mice (Figure 4e). Thus, memory $\mathrm{CD} 4^{+} \mathrm{T}$ cells deficient in IL-17A failed to mediate cross-protection and this failure was not due to any defects in activation or expansion of donor $\mathrm{CD} 4{ }^{+} / \mathrm{IL}-17 \mathrm{~A}^{-1-}$ cells in adoptive hosts. Taken together, these results show that cross-protection by memory $\mathrm{CD} 4^{+}$ $\mathrm{T}$ cells is dependent on its ability to produce IL-17A.

\section{Memory $\mathrm{CD}^{+}{ }^{+} \mathrm{T}$ cells provide broad protection against pneumonia by different serotype strains}

We further investigated if memory $\mathrm{CD} 4{ }^{+} \mathrm{T}$ cells can provide broad protection against many different serotype strains prevalent in clinics. $\mathrm{CD} 4^{+} \mathrm{T}$ cells from naïve $\left(\mathrm{CD} 4^{+} \mathrm{Tn}\right)$ and P1121-immune $\left(\mathrm{CD} 4^{+} \mathrm{Tm}\right)$ mice were transferred to naïve mice, which were then challenged with $10^{7} \mathrm{CFU}$ of the homologous $S p$ strain P1121 (serotype 23F) or heterologous $S p$ stains including BAA659 (serotype 6A), 700671 (serotype 9V), and 700676 (serotype 14). Mice that received $\mathrm{CD}^{+}{ }^{+} \mathrm{Tm}$ cells had significantly fewer bacteria in the lungs compared with mice that received $\mathrm{CD} 4{ }^{+} \mathrm{Tn}$ after challenge with homologous P1121 or heterologous strains, indicating that memory CD ${ }^{+}$ $\mathrm{T}$ cells provide broad protection against lung infection by different serotype $S p$ strains (Figure 5a). Consistent with broad protection, we observed strong Th17 recall responses to various serotype strains in P1121 immune mice; $\sim 20 \%$ of lung CD $4^{+}$ $\mathrm{T}$ cells from P1121-immune mice expressed IL-17A on day 7 after heterologous challenge, compared with $\sim 2 \%$ from 
a

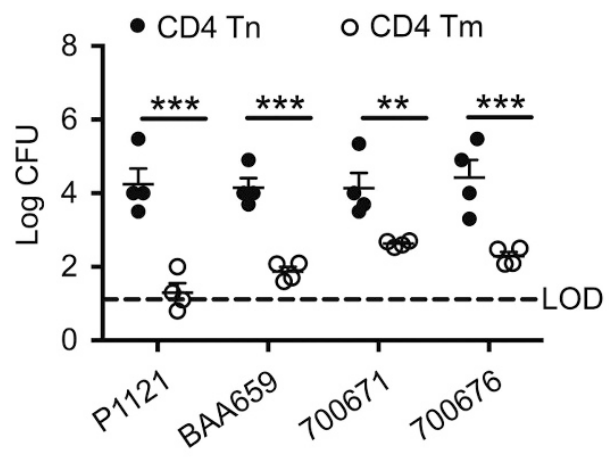

c

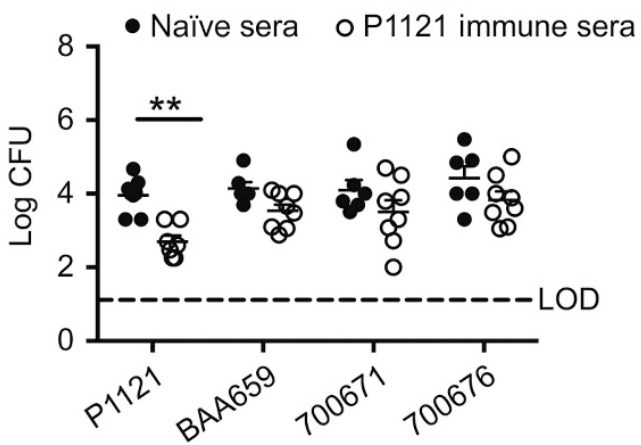

b
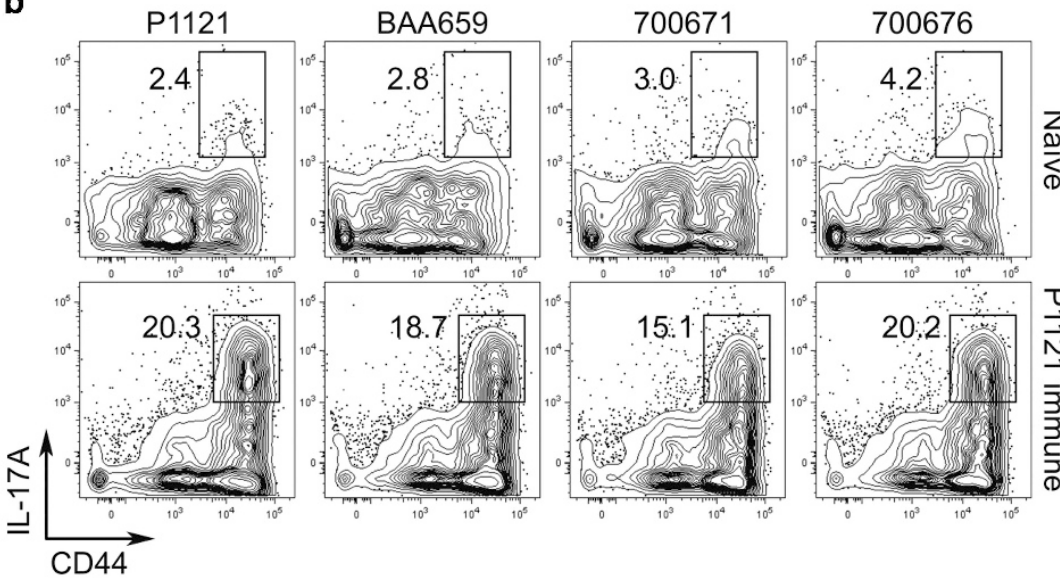

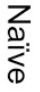
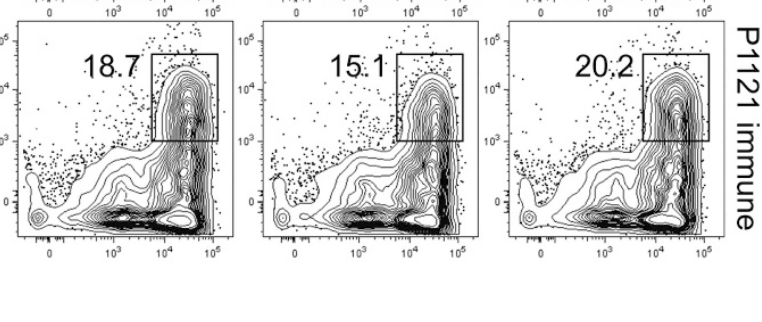

Figure 5 Streptococcus pneumoniae (Sp)-specific memory CD4 T cells provide broad protection against lung infection by different clinic Sp isolates. (a and b) CD4 T cells from naïve (CD4 ${ }^{+}$Tn, filled circle) or P1121-immunized mice (CD4 ${ }^{+}$Tm, open circle) were transferred into naïve B6 mice, which were then challenged with different Sp serotype strains. (a) Bacterial loads in lung homogenate on day 2 after challenge. (b) Interleukin-17A (IL-17A) production by CD4 ${ }^{+}$T cells following stimulation with heat-killed P1121, BAA659, 700671, and 700676. (c and d) Mice received sera from naïve (naïve sera) or P1121-immunized (P1121-immune sera) were infected with different Sp clinic isolates. (c) Bacterial loads in lung homogenate on day 2 after challenge. (d) Serum immunoglobulin G (IgG) against different Sp strains (whole bacteria) from P1121-immune mice (filled symbols), and against P1121 from naive mice (open circle). Tm, memory $\mathrm{T}$; Tn, naïve $\mathrm{T}$.

unimmunized mice infected with these strains (Figure $5 \mathbf{b}$ ). In contrast, transfer of P1121-immune sera protected against homologous P1121 challenge but resulted in no significant reduction of bacteria in the lung of mice challenged with heterologous strains (Figure 5c). P1121-immune sera had very low levels of crossreactive immunoglobulin G against BAA659, 700671, or 700676, which might account for the limited protection from P1121-immune sera (Figure 5d). Thus, CD ${ }^{+}$ T cells but not sera from P1121-immune mice conferred broad protection against pneumonia caused by various serotype $S p$ strains.

\section{DISCUSSION}

Extensive studies of $S p$-host interactions have focused on $S p$ colonization of the upper RT using the model of defined nasal infection. Although colonization is asymptomatic, it induces a rapid infiltration of neutrophils, followed by recruitment of monocytes/macrophages that is sustained until Sp colonization is cleared within 1-2 months. Although serotype-specific antibodies are generated, natural clearance of $S p$ colonization is not dependent on antibodies, but instead is reliant on monocytes/macrophages and Th17 CD ${ }^{+}$T cells. ${ }^{19}$ On the other hand, capsule-specific antibodies induced by PCV have an important role in reducing the carriage rate over time in a serotype-specific manner. ${ }^{17}$ It is not known whether this is the result of immune-mediated protection against colonization in vaccine recipients or the result of reduced circulation of these serotypes through herd immunity. Introduction of PCV has led to a substantial decrease in pneumonia caused by $S p$, which is thought to result from blockage of the initial step of colonization at the upper RT. It is not known if antibodies induced by PCV actually provide protective immunity in the lungs once $S p$ has gained entry and established infection in the lungs. In hosts that have weakened natural airway defenses such as following IAV infection, $S p$ may gain access and infect lungs directly resulting in rapid pneumonia without prior colonization of the upper RT. In this case, serotype-specific antibody induced by PCV may not be effective in preventing establishment of infection, and protection may require immune mechanisms other than capsule-specific antibodies that can attenuate infection in the lungs. Furthermore, increasing cases of $S p$ pneumonia are caused by replacement strains whose 
capsular polysaccharides are not included in PCV. Thus, understanding immune mechanisms of protection against $\mathrm{Sp}$ in the lungs is critical for developing effective vaccines that are broadly protective against bacterial pneumonia.

In this study, we used the murine pneumonia model where $S p$ is directly introduced into the lungs while bypassing the initial colonization step, ${ }^{21,23,30}$ and focused on immune mechanisms of cross-protection against lung infection. We tested the possibility of inducing heterologous protection against pneumonia by prior colonization, immunization with heat-inactivated $S p$ and prior sublethal lung infection. Our results showed that prior lung infection induced the highest level of protection, better than prior colonization and killed $S p$ immunization (Figure 1). Our results further revealed robust primary and recall Th17 responses localized in the lung mucosa in response to Sp lung infection, whereas only weak Th17 responses were induced by prior colonization, or heatinactivated $S p$ immunization (Supplementary Figure S4). Furthermore, our results showed that transfer of purified memory $\mathrm{CD}^{+}{ }^{+} \mathrm{T}$ cells protected against pneumonia in an IL17-dependent manner. We also tested the ability of memory $\mathrm{CD}^{+} \mathrm{T}$ cells to provide broad protection against clinical strains of different serotypes. While these clinical strains did not cause lethal pneumonia in mice (data not shown), there was significant reduction (100-1,000-fold) of bacterial loads in the lungs by memory CD $4{ }^{+}$T cells against all four serotype strains tested. Taken together, these results show that induction of localized Th17 response in the lungs by mucosal immunization is critical for cross-protection against $S p$ pneumonia.

Our results suggest that conserved $S p$ protein antigens might be vaccine candidates to offer broad protection by inducing strong memory Th17 response in the lungs. Moffitt et al. ${ }^{32}$ have identified two new pneumococcal proteins (SP2108 and SP0148) that induce Th17 responses and protection against colonization. It remains to be determined if these antigens can induce protective immunity against lung infection. Other conserved $S p$ proteins, such as pneumococcal surface antigen A, pneumolysin, pneumolysoid PdT, and histidine triad proteins have been used with potent adjuvants as subunit vaccines that protect mice against pneumonia. ${ }^{24,26,27,29,33}$ Although these studies have not directly tested the role of memory Th17 cells in protection against pneumonia, these findings together with our results support the notion that Th17-inducing conserved $S p$ protein antigens might be vaccine candidates capable of conferring broad protection by inducing strong memory Th17 responses in the lungs.

Following a primary $S p$ infection, the $\mathrm{CD} 4{ }^{+} \mathrm{T}$-cell response consists of a mix of weak Th1 and dominant Th17 responses, and a weak $\mathrm{CD} 8{ }^{+} \mathrm{T}$-cell response in the lungs. Surprisingly, the recall response in immune mice was exclusively by Th17 CD $4^{+}$ $\mathrm{T}$ cells with minimal contribution by $\mathrm{Th} 1 \mathrm{CD}^{+}$or $\mathrm{CD} 8{ }^{+}$ $\mathrm{T}$ cells. Previous studies have shown that IFN- $\gamma^{-/-}$mice quickly succumb to $S p$ lung infection, indicating an important role for IFN- $\gamma$ in controlling a primary lung infection. ${ }^{34} \mathrm{NKT}$ cells and neutrophils are the major source of IFN- $\gamma$ for the early control of a primary lung infection. ${ }^{35-38}$ Upregulation of ifng gene expression in the lung tissue is observed in $S p$-immune mice challenged with the homologous strains, but the source and requirement of IFN- $\gamma$ has not been identified. ${ }^{39}$ Our studies specifically examined the role of IFN- $\gamma$ produced by memory $\mathrm{T}$ cells in protection against reinfection. Our results showed that the levels of IFN- $\gamma$-secreting $\mathrm{CD} 4{ }^{+}$and $\mathrm{CD} 8{ }^{+} \mathrm{T}$ cells in immune mice challenged with T4 (P1121-T4) were similar at day 2, and even lower at day 7 compared with unimmunized mice infected with T4 (Supplementary Figure S5). Furthermore, in vivo blockade of IL-17A abrogated protective immunity conferred by adoptively transferred immune $\mathrm{CD}^{+} \mathrm{T}$ cells (Figure 4), while in vivo blockade of IFN- $\gamma$ did not (Supplementary Figure S6). Taken together, these results indicate that Th1 and IFN- $\gamma$ have a minimal role in protective immunity conferred by memory $\mathrm{CD} 4{ }^{+} \mathrm{T}$ cells against reinfection, although IFN- $\gamma$ has an important role in early control of primary infections. ${ }^{34-38}$ Th17 and Th1 responses are known to inter-regulate each other, and it remains to be determined if Th1 recall response is suppressed by a strong Th17 response or by other factors in this setting. Our data clearly demonstrated a critical role for Th17 cells, but it remains possible that the "balance" of Th17 and Th1 cells elicited following vaccination may be critical for generating effective protective immunity that promotes bacterial clearance and reduced immunopathology.

A combination of PCV with vaccines inducing Th17 memory $\mathrm{T}$ cells in the lungs may offer the best protection against bacterial pneumonia as they act at two distinct steps and by different immune mechanisms, antibodies blocking colonization at the upper RT while Th17 cells attenuating infection in the lungs.

\section{METHODS}

Animals. Female C57BL/6 and B6-Ly5.2/Cr (CD45.1) mice (6-8 weeks old) were purchased from National Cancer Institute (Fredericksburg, MD). IL-17A-deficient (IL-17 knockout (KO)) mice were originally developed by Dr Yoichiro Iwakura ${ }^{40}$ and provided by Dr David Artis (Weill Cornell Medical College, New York, NY). All animal experiments were performed in accordance with The University of Pennsylvania Institutional Animal Care and Use Committee protocols.

Pathogens and infections. Sp strain P1121 (serotype 23F) ${ }^{41}$ and TIGR4 (serotype 4) were used as described previously. ${ }^{42,43}$ Sp strain BAA659 (serotype 6A), 700671 (serotype 9V), 700676 (serotype 14) were gifts from Dr Irving Nachamkin (Department of Pathology and Laboratory Medicine, University of Pennsylvania, Philadelphia, PA). All $S p$ strains were grown in tryptic soy broth or agar plates as described. ${ }^{42}$ For lung infections, mice were anesthetized by intraperitoneal injections with $100 \mu \mathrm{l}$ ketamine/xylazine $\left(100 \mathrm{mg} / 3.8 \mathrm{mg} \mathrm{kg}^{-1}\right)$ and inoculated with $30 \mu \mathrm{l}$ of $S p$ suspensions $\left(10^{6}-10^{7} \mathrm{CFU}\right)$ intranasally. For colonization, unanesthetized mice were inoculated intranasally with $10 \mu \mathrm{l}$ of bacterial suspension. Mice were observed for clinical signs of morbidity by monitoring body weights daily. BALF, lung homogenates were prepared as described, ${ }^{22}$ and $10 \mu \mathrm{l}$ of serial dilutions were plated in triplicate. The limit of detection for bacteria in lavage or lung homogenate was $10 \mathrm{CFU} \mathrm{ml}{ }^{-1}$.

Flow cytometry and luminex assay. Lymphocytes from the lungs and spleens were isolated and stained as described previously. ${ }^{44,45}$ For intracellular staining, cells were stimulated with heat-killed bacteria 
$\left(65^{\circ} \mathrm{C}\right.$ for $30 \mathrm{~min}$ ) at indicated multiplicity of infection for $16 \mathrm{~h}$ with Golgi Plug/Stop added at the last $4 \mathrm{~h}$, and then stained as described. ${ }^{44}$ Millipore Multiplex Kits (MPXMCYTO-70k-16) were used for detection of cytokines and chemokines from BALF.

Histology. Lung sections were prepared as described previously. ${ }^{46}$ Apoptosis was analyzed using In Situ Cell Death Detection Kit, fluorescein (Roche, Indianapolis, IN). Sections were processed and stained by the Cardiology Histology Core and Cancer Histology Core at the University of Pennsylvania.

Adoptive transfer and in vivo antibody treatment. Total $\mathrm{T}$ cells or $\mathrm{CD}^{+}{ }^{+} \mathrm{T}$ cells from the spleens and lungs were enriched using MACS Pan T-cell Isolation Kit II or CD4 microbeads (Miltenyi Biotec, San Diego, CA), ${ }^{47}$ and assayed for a purity ( $>95 \%$ ) by flow cytometry. Purified T cells, non-T cells or CD4 T cells $\left(3-5 \times 10^{6}\right)$ were transferred intravenously into naive mice one day before $S p$ challenge.

In vivo IL-17 or IFN- $\gamma$ neutralization was achieved as previously described with slight modifications. ${ }^{48-50}$ Mice received $180 \mu \mathrm{g}$ intraperitoneally and $20 \mu \mathrm{g}$ intranasally of anti-IL-17 (clone 17F3) or IFN- $\gamma$ antibody (clone XMG1.2) on days $-1,0$, and 1 following $S p$ challenge. Neutralizing efficiency was verified using Luminex for BALF and ELISA (enzyme-linked immunosorbent assay) for serum on days 2 and 7 after infection.

Statistical analyses. Unpaired, one-tailed, Student's $t$-tests were used to calculate statistical significance between two groups and one-way analysis of variance was used for comparison of multiple groups followed by Bonferroni correction unless stated otherwise. $P$ values are depicted as follows: ${ }^{\star} P<0.05 ;{ }^{* *} P<0.01 ;{ }^{* * *} P<0.001$; ${ }^{* * * *} P<0.0001$ and ${ }^{\text {ns }} P>0.05$. A $P$ value $\leq 0.05$ was considered significant.

SUPPLEMENTARY MATERIAL is linked to the online version of the paper at http://www.nature.com/mi

\section{ACKNOWLEDGMENTS}

We thank members of the Shen lab for technical support and critical discussions, and acknowledge the help from Tianying Jiang and Lan Cheng for histology, Aoife Roche for ELISA, Dr Irving Nachamkin for various $\mathrm{Sp}$ strains, and Dr Steven Furyk for careful editing of the manuscript. This work was supported by NIH grants AI083022 and Al095740 (to HS) and AI038446 and Al105168 (to JNW).

\section{DISCLOSURE}

The authors declared no conflict of interest.

c) 2017 Society for Mucosal Immunology

\section{REFERENCES}

1. Shak, J.R., Vidal, J.E. \& Klugman, K.P. Influence of bacterial interactions on pneumococcal colonization of the nasopharynx. Trends Microbiol. 21, 129-135 (2013).

2. Bogaert, D., De Groot, R. \& Hermans, P.W. Streptococcus pneumoniae colonisation: the key to pneumococcal disease. Lancet Infect. Dis. 4, 144-154 (2004).

3. Tigoi, C.C. et al. Rates of acquisition of pneumococcal colonization and transmission probabilities, by serotype, among newborn infants in Kilifi District, Kenya. Clin. Infect. Dis. 55, 180-188 (2012).

4. Mosser, J.F. et al. Nasopharyngeal carriage and transmission of Streptococcus pneumoniae in American Indian households after a decade of pneumococcal conjugate vaccine use. PLoS One 9, e79578 (2014).

5. Kadioglu, A., Weiser, J.N., Paton, J.C. \& Andrew, P.W. The role of Streptococcus pneumoniae virulence factors in host respiratory colonization and disease. Nat. Rev. Microbiol. 6, 288-301 (2008).

6. Fineberg, H.V. Pandemic preparedness and response-lessons from the H1N1 influenza of 2009. N. Engl. J. Med. 370, 1335-1342 (2014).
7. Chien, Y.W., Klugman, K.P. \& Morens, D.M. Bacterial pathogens and death during the 1918 influenza pandemic. N. Engl. J. Med. 361, 2582-2583 (2009).

8. Eberhardt, A. et al. Attachment of capsular polysaccharide to the cell wall in Streptococcus pneumoniae. Microbial Drug Resist. 18, 240-255 (2012).

9. Hammerschmidt, S., Wolff, S., Hocke, A., Rosseau, S., Muller, E. \& Rohde, M. Illustration of pneumococcal polysaccharide capsule during adherence and invasion of epithelial cells. Infect. Immun. 73, 4653-4667 (2005).

10. Pilishvili, T. et al. Sustained reductions in invasive pneumococcal disease in the era of conjugate vaccine. J. Infect. Dis. 201, 32-41 (2010).

11. Poehling, K.A. et al. Invasive pneumococcal disease among infants before and after introduction of pneumococcal conjugate vaccine. JAMA 295, 1668-1674 (2006).

12. Hanage, W.P. Serotype replacement in invasive pneumococcal disease: where do we go from here?. J. Infect. Dis. 196, 1282-1284 (2007).

13. Weinberger, D.M., Malley, R. \& Lipsitch, M. Serotype replacement in disease after pneumococcal vaccination. Lancet 378, 1962-1973 (2011).

14. O'Brien, K.L. et al. Burden of disease caused by Streptococcus pneumoniae in children younger than 5 years: global estimates. Lancet 374, 893-902 (2009).

15. van der Poll, T. \& Opal, S.M. Pathogenesis, treatment, and prevention of pneumococcal pneumonia. Lancet 374, 1543-1556 (2009).

16. Nurhonen, M. \& Auranen, K. Optimal serotype compositions for Pneumococcal conjugate vaccination under serotype replacement. PLOS Comput. Biol. 10, e1003477 (2014).

17. Moffitt, K.L. \& Malley, R. Next generation pneumococcal vaccines. Curr. Opin. Immunol. 23, 407-413 (2011).

18. Malley, R., Trzcinski, K., Srivastava, A., Thompson, C.M., Anderson, P.W. \& Lipsitch, M. CD4 + T cells mediate antibody-independent acquired immunity to pneumococcal colonization. Proc. Natl. Acad. Sci. USA 102, 4848-4853 (2005).

19. Zhang, Z., Clarke, T.B. \& Weiser, J.N. Cellular effectors mediating Th17dependent clearance of pneumococcal colonization in mice. J. Clin. Invest. 119, 1899-1909 (2009).

20. Malley, R. et al. Antibody-independent, interleukin-17A-mediated, crossserotype immunity to pneumococci in mice immunized intranasally with the cell wall polysaccharide. Infect. Immun. 74, 2187-2195 (2006).

21. Cohen, J.M., Khandavilli, S., Camberlein, E., Hyams, C., Baxendale, H.E. \& Brown, J.S. Protective contributions against invasive Streptococcus pneumoniae pneumonia of antibody and Th17-cell responses to nasopharyngeal colonisation. PLoS One 6, e25558 (2011).

22. Wilson, R., Cohen, J.M., Jose, R.J., de Vogel, C., Baxendale, H. \& Brown, J.S. Protection against Streptococcus pneumoniae lung infection after nasopharyngeal colonization requires both humoral and cellular immune responses. Mucosal Immunol. 8, 627-639 (2014).

23. Cohen, J.M., Wilson, R., Shah, P., Baxendale, H.E. \& Brown, J.S. Lack of cross-protection against invasive pneumonia caused by heterologous strains following murine Streptococcus pneumoniae nasopharyngeal colonisation despite whole cell ELISAs showing significant cross-reactive IgG. Vaccine 31, 2328-2332 (2013).

24. Ferreira, D.M. et al. Characterization of protective mucosal and systemic immune responses elicited by pneumococcal surface protein PspA and PspC nasal vaccines against a respiratory pneumococcal challenge in mice. Clin. Vaccine Immunol. 16, 636-645 (2009).

25. Lu, Y.J. et al. GMP-grade pneumococcal whole-cell vaccine injected subcutaneously protects mice from nasopharyngeal colonization and fatal aspiration-sepsis. Vaccine 28, 7468-7475 (2010).

26. Zhong, W. et al. Mucosal immunization with caseinolytic protease Xelicited cross-protective immunity against pneumococcal infection in mice. Exp. Biol. Med. (Maywood) 237, 694-702 (2012).

27. Lima, F.A. et al. Controlled inflammatory responses in the lungs are associated with protection elicited by a pneumococcal surface protein A-based vaccine against a lethal respiratory challenge with Streptococcus pneumoniae in mice. Clin. Vaccine Immunol. 19, 1382-1392 (2012).

28. Liu, Y. et al. Mucosal immunization with recombinant fusion protein DnaJDeltaA146Ply enhances cross-protective immunity against Streptococcus pneumoniae infection in mice via interleukin 17A. Infect. Immun. 82, 16661675 (2014)

29. Salcedo-Rivillas, C. et al. Pertussis toxin improves immune responses to a combined pneumococcal antigen and leads to enhanced protection 
against Streptococcus pneumoniae. Clin. Vaccine Immunol. 21, 972-981 (2014).

30. Richards, L., Ferreira, D.M., Miyaji, E.N., Andrew, P.W. \& Kadioglu, A. The immunising effect of pneumococcal nasopharyngeal colonisation; protection against future colonisation and fatal invasive disease. Immunobiology 215, 251-263 (2010).

31. McCool, T.L. \& Weiser, J.N. Limited role of antibody in clearance of Streptococcus pneumoniae in a murine model of colonization. Infect. Immun. 72, 5807-5813 (2004).

32. Moffitt, K.L. et al. $T(H) 17$-based vaccine design for prevention of Streptococcus pneumoniae colonization. Cell Host Microbe 9, 158165 (2011).

33. Xu, Q., Surendran, N., Verhoeven, D., Klapa, J., Ochs, M. \& Pichichero, M.E. Trivalent pneumococcal protein recombinant vaccine protects against lethal Streptococcus pneumoniae pneumonia and correlates with phagocytosis by neutrophils during early pathogenesis. Vaccine 33 , 9931000 (2015).

34. Weber, S.E., Tian, H. \& Pirofski, L.A. CD8 + cells enhance resistance to pulmonary serotype 3 Streptococcus pneumoniae infection in mice. J. Immunol. 186, 432-442 (2011).

35. Nakamatsu, M. et al. Role of interferon-gamma in Valpha14 + natural killer Tcell-mediated host defense against Streptococcus pneumoniae infection in murine lungs. Microbes Infect. 9, 364-374 (2007).

36. Kinjo, Y. et al. Invariant natural killer $T$ cells recognize glycolipids from pathogenic Gram-positive bacteria. Nat. Immunol. 12, 966-974 (2011).

37. Yamada, M. et al. Interferon-gamma production by neutrophils during bacterial pneumonia in mice. Am. J. Respir. Crit. Care Med. 183, 13911401 (2011).

38. Ivanov, S. et al. Key role for respiratory CD103(+) dendritic cells, IFN-gamma, and IL-17 in protection against Streptococcus pneumoniae infection in response to alpha-galactosylceramide. J. Infect. Dis. 206, 723-734 (2012).
39. Marques, J.M. et al. Protection against Streptococcus pneumoniae serotype 1 acute infection shows a signature of Th17- and IFNgamma-mediated immunity. Immunobiology 217, 420-429 (2012).

40. Nakae, S., Nambu, A., Sudo, K. \& Iwakura, Y. Suppression of immune induction of collagen-induced arthritis in IL-17-deficient mice. J. Immunol. 171, 6173-6177 (2003).

41. McCool, T.L., Cate, T.R., Moy, G. \& Weiser, J.N. The immune response to pneumococcal proteins during experimental human carriage. J. Exp. Med. 195, 359-365 (2002).

42. Davis, K.M., Akinbi, H.T., Standish, A.J. \& Weiser, J.N. Resistance to mucosal lysozyme compensates for the fitness deficit of peptidoglycan modifications by Streptococcus pneumoniae. PLoS Pathog. 4, e1000241 (2008).

43. Tettelin, $\mathrm{H}$. et al. Complete genome sequence of a virulent isolate of Streptococcus pneumoniae. Science 293, 498-506 (2001).

44. Pearce, E.L. \& Shen, H. Generation of CD8 Tcell memory is regulated by IL12. J. Immunol. 179, 2074-2081 (2007).

45. Sauer, K.A., Scholtes, P., Karwot, R. \& Finotto, S. Isolation of CD4 + Tcells from murine lungs: a method to analyze ongoing immune responses in the lung. Nat. Protoc. 1, 2870-2875 (2006).

46. Tian, Y. et al. Regulation of lung endoderm progenitor cell behavior by miR302/367. Development 138, 1235-1245 (2011).

47. Dispirito, J.R. \& Shen, H. Histone acetylation at the single-cell level: a marker of memory $\mathrm{CD} 8+\mathrm{T}$ cell differentiation and functionality. J. Immunol. 184, 4631-4636 (2010).

48. Gonzalez-Lombana, C. et al. IL-17 mediates immunopathology in the absence of IL-10 following Leishmania major infection. PLoS Pathog. 9, e1003243 (2013).

49. Berger, H. et al. SOCS3 transactivation by PPARgamma prevents IL-17driven cancer growth. Cancer Res. 73, 3578-3590 (2013).

50. Cortez, V.S. et al. CRTAM controls residency of gut CD4 + CD8 + Tcells in the steady state and maintenance of gut CD4 + Th17 during parasitic infection. J. Exp. Med. 211, 623-633 (2014). 